Supporting Information for:

\title{
Reduced Phosphomolybdate Hybrids As Efficient Visible-Light Photocatalysts for $\mathrm{Cr}(\mathrm{VI})$ reduction
}

Lin Hou, Yaqi Zhang, Yuanyuan Ma*, Yali Wang, Zhifei Hu, Yuanzhe

$$
\mathrm{Gao}^{*} \text {, Zhangang Han* }
$$

Hebei Key Laboratory of Organic Functional Molecules, National Demonstration Center for Experimental Chemistry Education, College of Chemistry and Material Science, Hebei Normal University, Shijiazhuang, Hebei 050024, People’s Republic of China 


\section{CONTENTS:}

Figure S1: UV absorption spectra of different concentrations of $\mathrm{Cr}(\mathrm{VI})$ solution (mass concentration from the inside out were $0.0136 \times 10^{-3}, 0.0272 \times 10^{-3}, 0.0544 \times 10^{-3}$, $\left.0.0680 \times 10^{-3}, 0.1088 \times 10^{-3}, 0.1360 \times 10^{-3}, 0.2720 \times 10^{-3} \mathrm{M}\right)$. The graph shows the relationship between the mass concentration of $\mathrm{Cr}(\mathrm{VI})$ solution and absorbance at a wavelength of $352 \mathrm{~nm}$

Figure S2: a) The 2D structure linked through H-bonding interactions; b) The $\pi$ - $\pi$ interaction among layers (the different layered structures in different colors)

Figure S3: ORTEP view of the asymmetric unit in $\mathbf{2}$ showing the atom labeling scheme $(\#=2$-x, $1-\mathrm{y},-\mathrm{z})$. .3

Figure S4: ORTEP view of the asymmetric unit in $\mathbf{3}$ showing the atom labeling scheme (\# =-x,-y, $1-\mathrm{z})$ 4

Figure S5: IR spectra of crystals 1-3 (KBr pellets as background) .............................

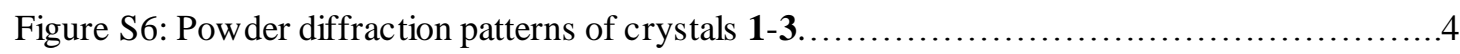

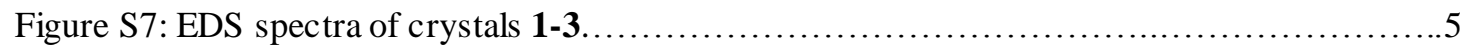

Figure S8: Cyclic voltammograms of (a) 1-GCE (GCE = Glass Carbon Electrode), (b) 2-GCE, and

(c) 3-GCE in the $0.5 \mathrm{M} \mathrm{H}_{2} \mathrm{SO}_{4}$ solution at different scan rates (from inner to outer: 20, $50,80,110$, and $140 \mathrm{mV} \cdot \mathrm{s}^{-1}$ ). Potentials $v s . \mathrm{Ag} / \mathrm{AgCl}$ (inset plots: the dependence of anodic peak II currents against scan rates)........................................

Figure S9: Photoreduction of $\mathrm{Cr}(\mathrm{VI})$ catalyzed by different dosages of $\mathbf{1}$ under a 10W white-light irradiation at room temperature.

Figure S10: A view showing the photoreduction of $\mathrm{Cr}(\mathrm{VI})$ when zinc oxide is used as catalyst under visible-light irradiation at room temperature ......................................6

Figure S11: Comparison of XRD patterns of the fresh 1-3 and after catalysis..................

Figure S12: Comparison of IR spectra of the fresh $\mathbf{1}, \mathbf{3}$ and after catalysis .......................

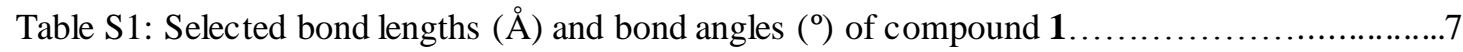

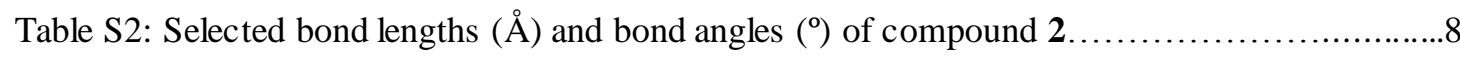

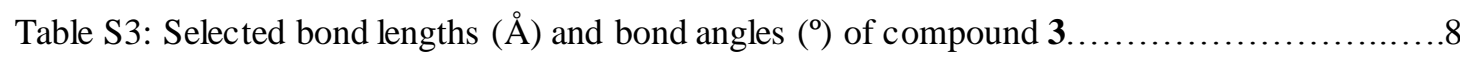

Table S4: BVS calculations of Mo, $\mathrm{P}$ and $\mathrm{Zn}$ centers in compounds 1-3.......................9

Table S5: Redox potentials (mV vs. Ag/AgCl) on I-I', II-II' and III-III' for 1-3 GCEs at the scan rate of $140 \mathrm{mV} \cdot \mathrm{s}^{-1}$ 


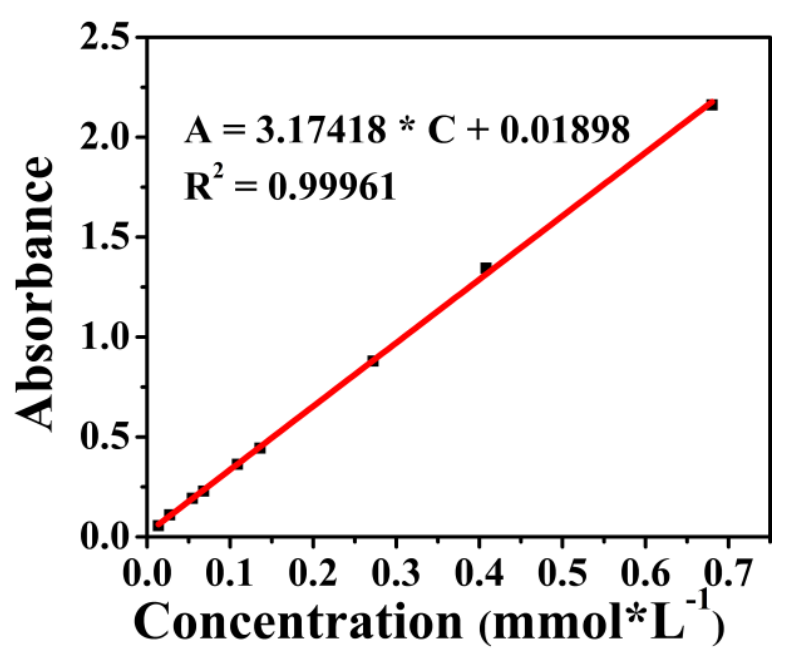

Figure S1 UV absorption spectra of different concentrations of $\mathrm{Cr}(\mathrm{VI})$ solution (mass concentration from the inside out were $0.0136 \times 10^{-3}, 0.0272 \times 10^{-3}, 0.0544 \times 10^{-3}, 0.0680 \times$ $\left.10^{-3}, 0.1088 \times 10^{-3}, 0.1360 \times 10^{-3}, 0.2720 \times 10^{-3} \mathrm{M}\right)$. The graph shows the relationship between the mass concentration of $\mathrm{Cr}(\mathrm{VI})$ solution and absorbance at a wavelength of 352 nm.

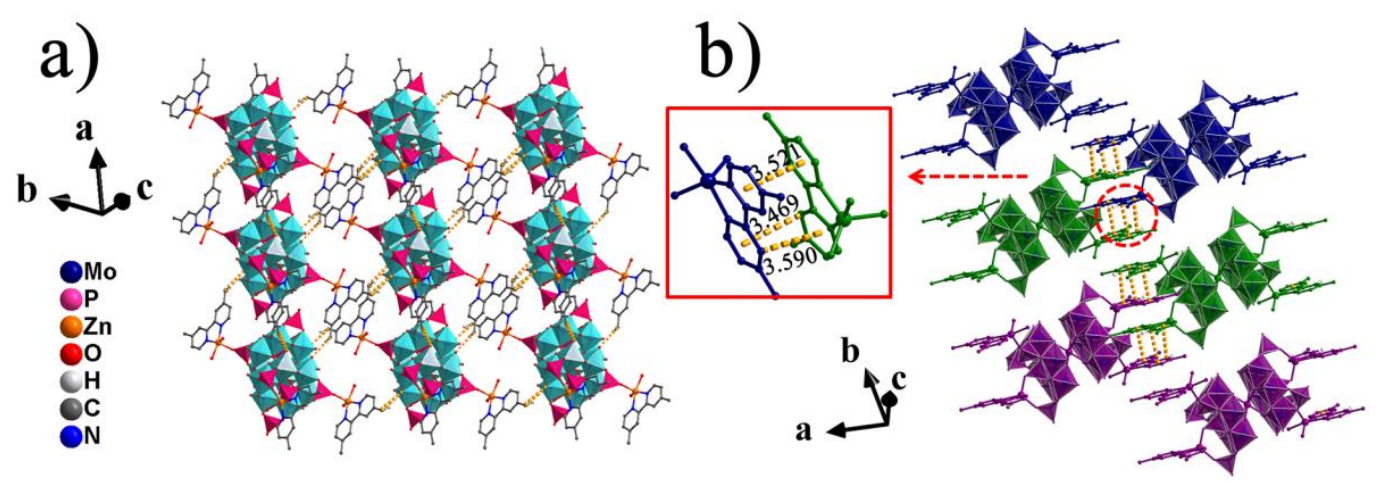

Figure S2 a) The 2D structure linked through H-bonding interactions; b) The $\pi-\pi$ interaction among layers (the different layered structures in different colors).

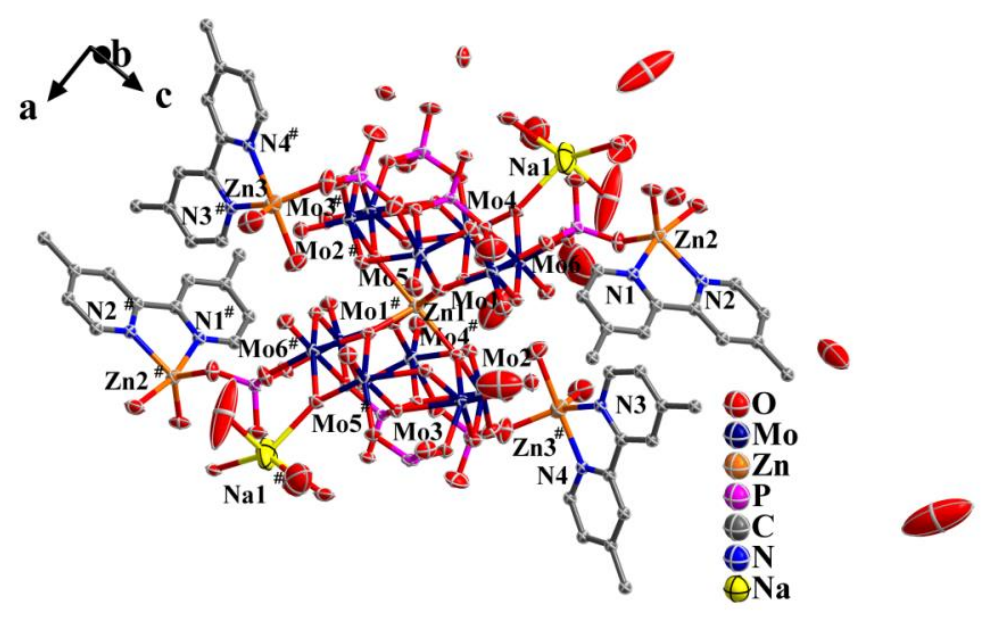

Figure S3 ORTEP view of the asymmetric unit in $\mathbf{2}$ showing the atom labeling scheme (\# = 2-x, $1-\mathrm{y},-\mathrm{z})$. 


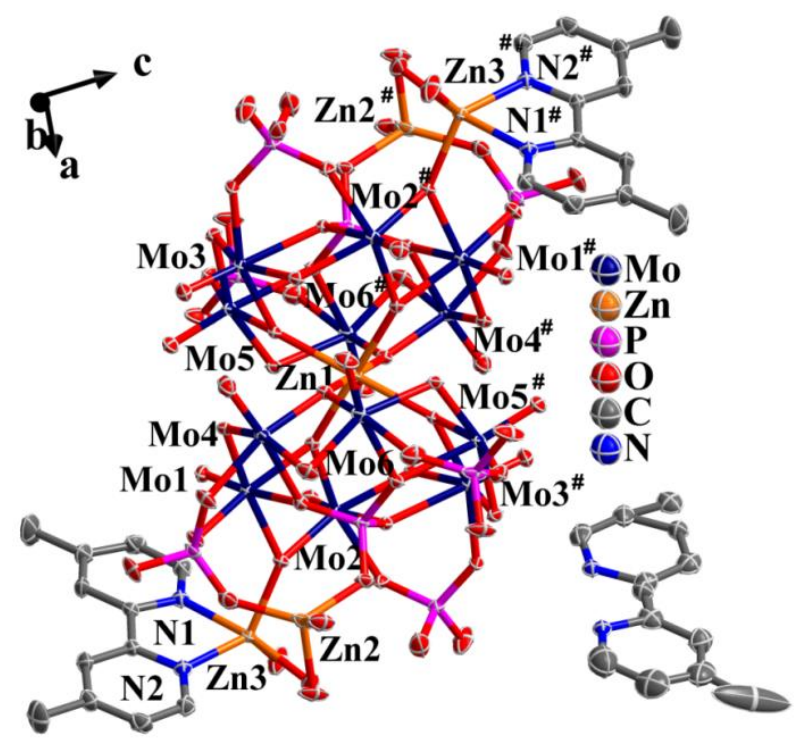

Figure S4 ORTEP view of the asymmetric unit in $\mathbf{3}$ showing the atom labeling scheme (\# =-x, -y, $1-z)$.

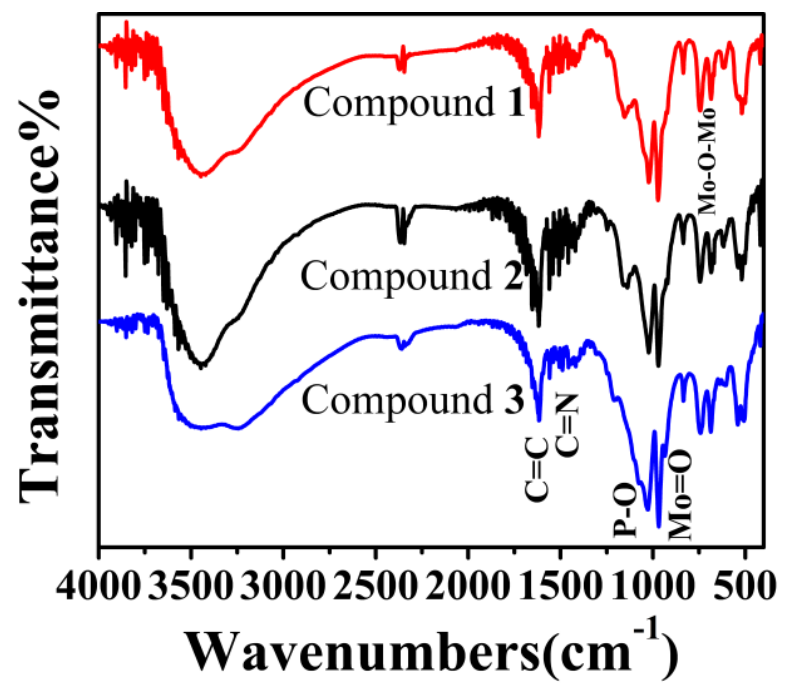

Figure S5 IR spectra of crystals 1-3 (KBr pellets as background).

a)

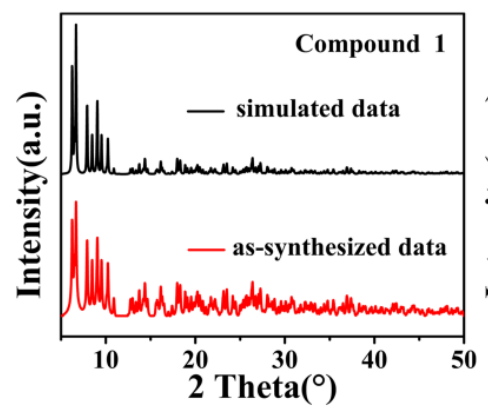

b)

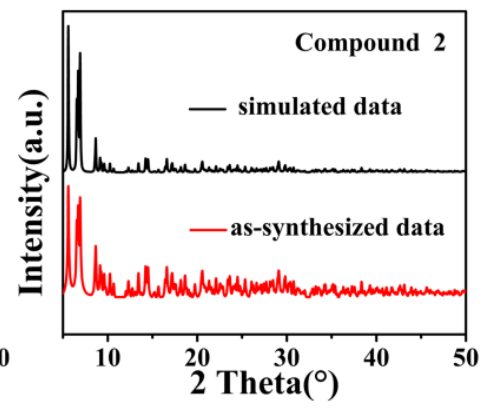

c)

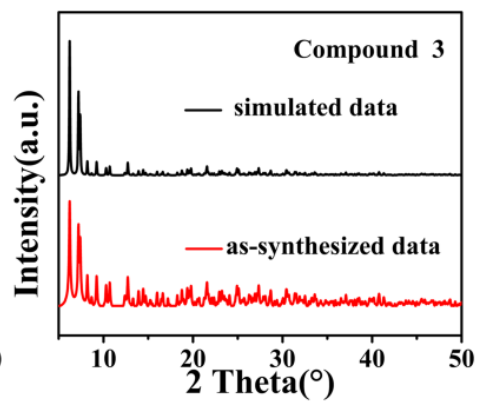

Figure S6 Powder diffraction patterns of crystals 1-3. 


\begin{tabular}{|c|c|c|c|}
\hline & Elements & Weight $\%$ & Atom \% \\
\hline \multirow{6}{*}{$\begin{array}{c}\text { Compound } \\
\mathbf{1}\end{array}$} & C K & 35.12 & 47.56 \\
\hline & $\mathrm{O} \mathrm{K}$ & 47.45 & 48.23 \\
\hline & P K & 3.30 & 1.73 \\
\hline & Zn K & 0.97 & 0.24 \\
\hline & Mo L & 13.16 & 2.23 \\
\hline & Sum & 100.00 & \\
\hline \multirow{7}{*}{$\begin{array}{c}\text { Compound } \\
2\end{array}$} & C K & 16.41 & 25.36 \\
\hline & $\mathrm{Na} \mathrm{K}$ & 0.51 & 0.41 \\
\hline & P K & 3.80 & 2.27 \\
\hline & Zn K & 5.23 & 1.49 \\
\hline & Mo L & 15.98 & 3.09 \\
\hline & $\mathrm{O} \mathrm{K}$ & 58.07 & 67.37 \\
\hline & Sum & 100.00 & \\
\hline \multirow{7}{*}{$\begin{array}{c}\text { Compound } \\
\mathbf{3}\end{array}$} & C K & 46.89 & 63.99 \\
\hline & $\mathrm{O} \mathrm{K}$ & 29.63 & 30.36 \\
\hline & P K & 3.43 & 1.81 \\
\hline & Zn K & 5.11 & 1.28 \\
\hline & Mo L & 14.93 & 2.55 \\
\hline & C K & 46.89 & 63.99 \\
\hline & Sum & 100.00 & \\
\hline
\end{tabular}
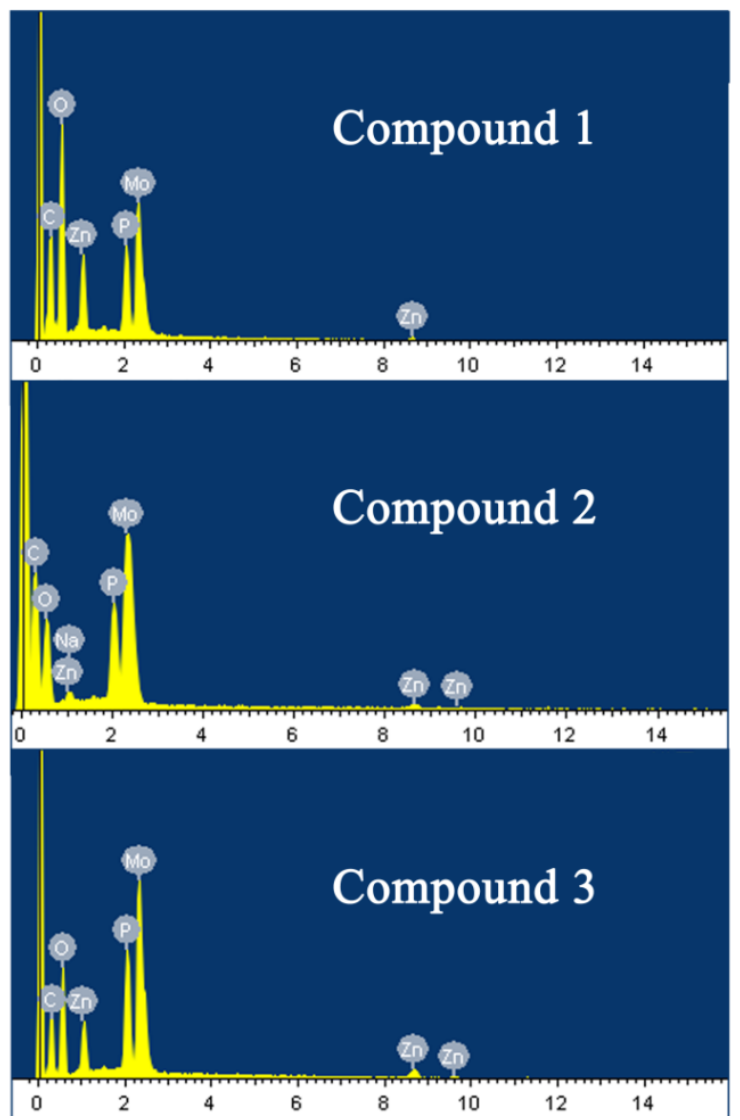

Figure S7 EDS spectra of crystals 1-3.

a)

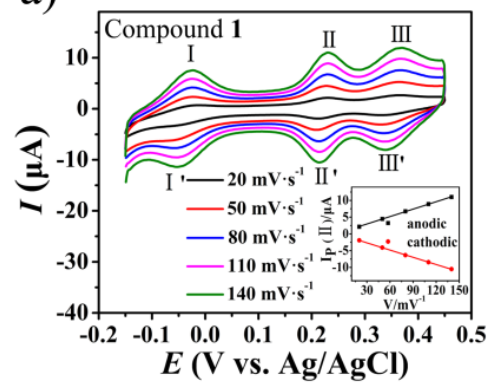

b)

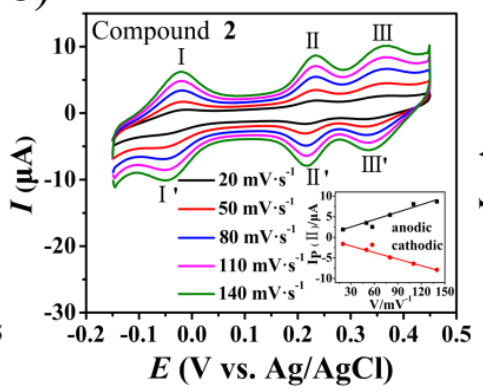

c)

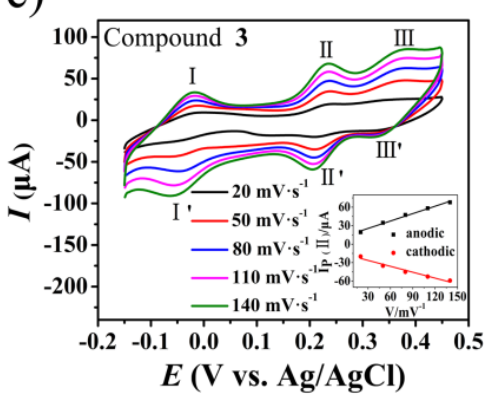

Figure S8 Cyclic voltammograms of (a) 1-GCE (GCE = Glass Carbon Electrode), (b) 2-GCE, and (c) 3-GCE in the $0.5 \mathrm{M} \mathrm{H}_{2} \mathrm{SO}_{4}$ solution at different scan rates (from inner to outer: 20, 50, 80, 110 , and $140 \mathrm{mV} \cdot \mathrm{s}^{-1}$ ). Potentials $v s . \mathrm{Ag} / \mathrm{AgCl}$ (inset plots: the dependence of anodic peak II currents against scan rates). 


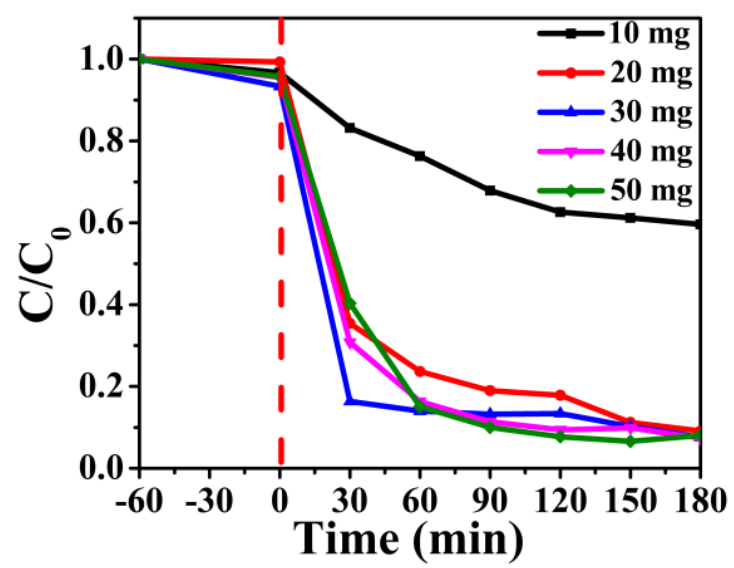

Figure S9 Photoreduction of $\mathrm{Cr}(\mathrm{VI})$ catalyzed by different dosages of $\mathbf{1}$ under a 10W white-light irradiation at room temperature.

To optimize the reaction parameters of the catalytic system, such as the influence of the dosage of catalyst, a series of reactions were carried out. And the absorption peaks of $\mathrm{Cr}(\mathrm{VI})$ ions distinctly decreased with increasing reaction time. When using $\mathbf{1}$ as the catalyst, Figure S9 showed that the conversion rates of $\mathrm{K}_{2} \mathrm{Cr}_{2} \mathrm{O}_{7}$ changed with the increase of the dosages of catalyst at room temperature. The conversion rate of $\mathrm{Cr}(\mathrm{VI})$ raised with the increasing of catalyst dosages, but when the amount of catalyst exceeds $20 \mathrm{mg}$, the rising trend decreased, and it only has a little effect on increasing catalytic activity by continuing raising the amount of catalyst. Therefore, 20 $\mathrm{mg}$ is chosen as the best dosage of catalyst in the principle of being more green and resource-saving.

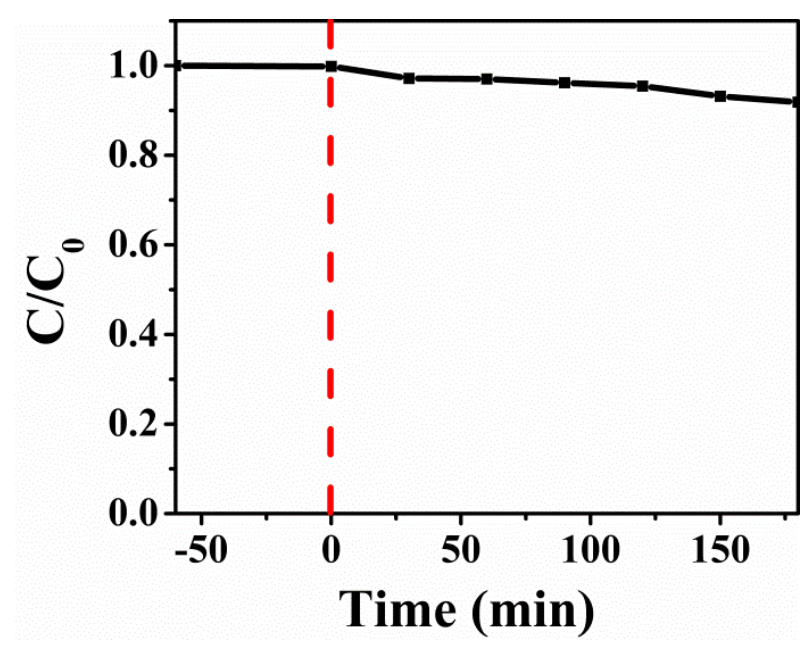

Figure S10 A view showing the photoreduction of $\mathrm{Cr}(\mathrm{VI})$ when zinc oxide is used as catalyst under visible-light irradiation at room temperature. 

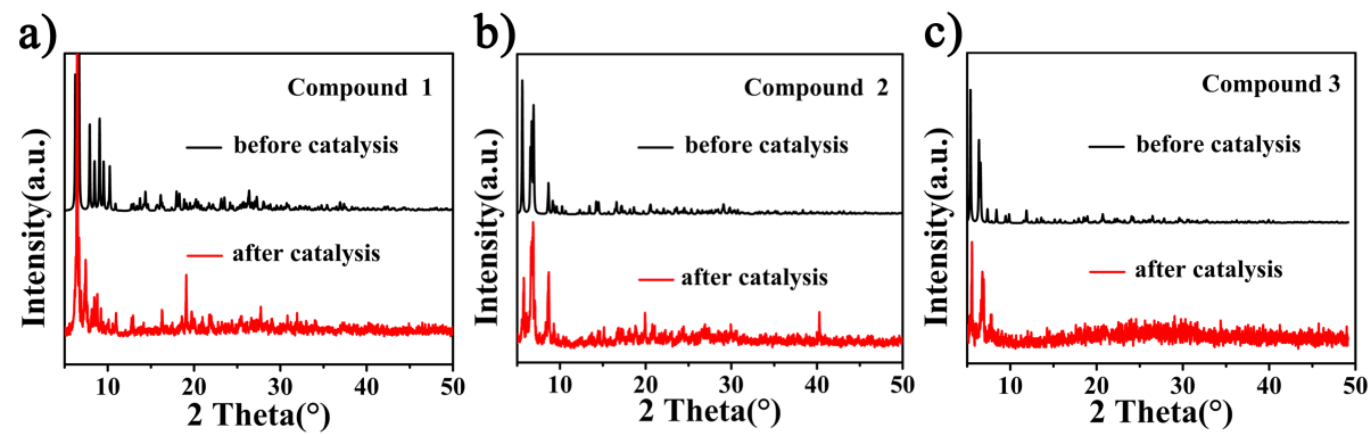

Figure S11 Comparison of XRD patterns of the fresh 1-3 and after catalysis.
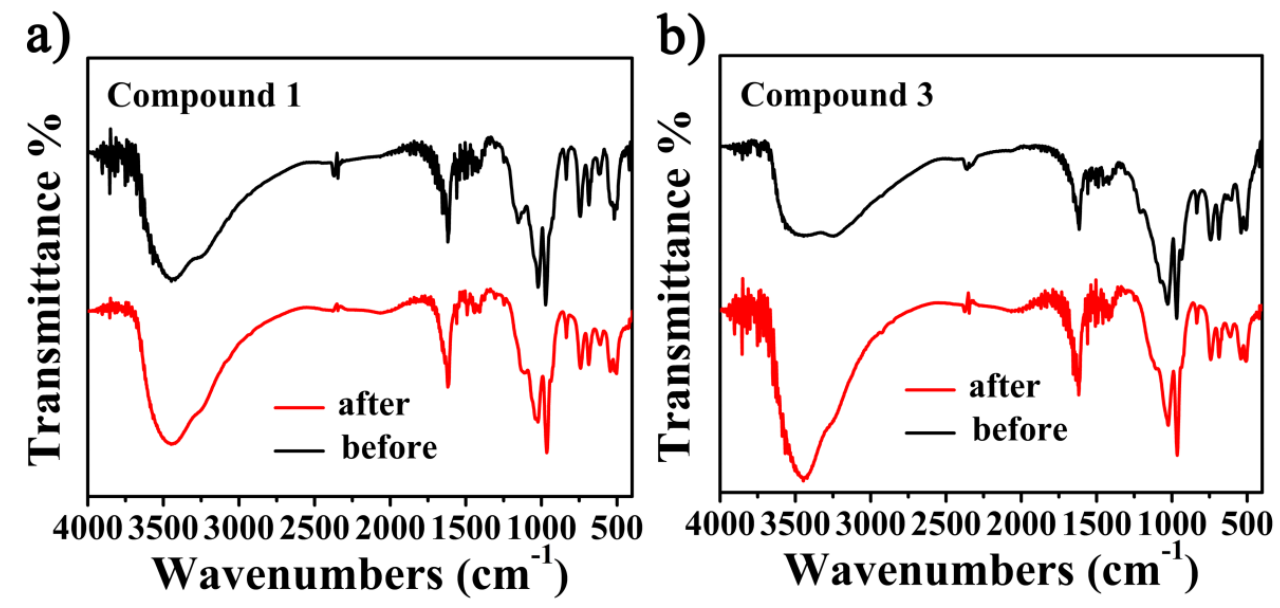

Figure S12 Comparison of IR spectra of the fresh $\mathbf{1}, \mathbf{3}$ and after catalysis.

Table S1 Selected bond lengths $(\AA)$ and bond angles $\left({ }^{\circ}\right)$ of compound $\mathbf{1}$.

\begin{tabular}{llllll}
\hline $\mathrm{Mo}(1)-\mathrm{O}(25)$ & $1.667(4)$ & $\mathrm{Mo}(5)-\mathrm{O}(26)$ & $1.679(4)$ & $\mathrm{P}(2)-\mathrm{O}(24)$ & $1.524(5)$ \\
$\mathrm{Mo}(1)-\mathrm{O}(10)$ & $1.950(4)$ & $\mathrm{Mo}(5)-\mathrm{O}(17)$ & $1.939(4)$ & $\mathrm{P}(2)-\mathrm{O}(23)$ & $1.506(5)$ \\
$\mathrm{Mo}(2)-\mathrm{O}(21)$ & $1.676(4)$ & $\mathrm{Mo}(6)-\mathrm{O}(11)$ & $1.672(4)$ & $\mathrm{P}(3)-\mathrm{O}(20)$ & $1.506(4)$ \\
$\mathrm{Mo}(2)-\mathrm{O}(15)$ & $1.932(4)$ & $\mathrm{Mo}(6)-\mathrm{O}(17)$ & $1.932(4)$ & $\mathrm{P}(4)-\mathrm{O}(13)$ & $1.504(4)$ \\
$\mathrm{Mo}(3)-\mathrm{O}(28)$ & $1.676(4)$ & $\mathrm{Zn}(1)-\mathrm{O}(2)$ & $2.136(4)$ & $\mathrm{Zn}(2)-\mathrm{N}(1)$ & $2.091(6)$ \\
$\mathrm{Mo}(3)-\mathrm{O}(10)$ & $1.945(4)$ & $\mathrm{Zn}(1)-\mathrm{O}(6)$ & $2.184(4)$ & $\mathrm{Zn}(2)-\mathrm{N}(2)$ & $2.124(5)$ \\
$\mathrm{Mo}(4)-\mathrm{O}(30)$ & $1.677(4)$ & $\mathrm{P}(1)-\mathrm{O}(9)$ & $1.518(4)$ & $\mathrm{Zn}(3)-\mathrm{N}(3)$ & $2.097(6)$ \\
$\mathrm{Mo}(4)-\mathrm{O}(15)$ & $1.930(4)$ & $\mathrm{P}(1)-\mathrm{O}(5)$ & $1.549(4)$ & $\mathrm{Zn}(3)-\mathrm{O}(3 \mathrm{~W})$ & $2.112(9)$ \\
$\mathrm{O}(25)-\mathrm{Mo}(1)-\mathrm{O}(10)$ & $104.8(2)$ & $\mathrm{O}(28)-\mathrm{Mo}(3)-\mathrm{O}(10)$ & $106.4(2)$ & $\mathrm{Na}(1)-\mathrm{P}(1)-\mathrm{Na}(2)$ & $78.26(5)$ \\
$\mathrm{O}(25)-\mathrm{Mo}(1)-\mathrm{O}(6)$ & $103.2(2)$ & $\mathrm{O}(28)-\mathrm{Mo}(3)-\mathrm{O}(16)$ & $96.4(2)$ & $\mathrm{O}(23)-\mathrm{Zn}(2)-\mathrm{N}(2)$ & $94.9(2)$ \\
$\mathrm{O}(25)-\mathrm{Mo}(1)-\mathrm{O}(1)$ & $95.5(2)$ & $\mathrm{O}(7)-\mathrm{Mo}(3)-\mathrm{Mo}(1)$ & $87.89(9)$ & $\mathrm{N}(1)-\mathrm{Zn}(2)-\mathrm{N}(2)$ & $77.9(2)$ \\
$\mathrm{O}(5)-\mathrm{Mo}(1)-\mathrm{Mo}(3)$ & $89.39(9)$ & $\mathrm{O}(9)-\mathrm{P}(1)-\mathrm{O}(5)$ & $113.3(2)$ & $\mathrm{O}(2 \mathrm{~W})-\mathrm{Zn}(3)-\mathrm{O}(3 \mathrm{~W})$ & $89.7(4)$ \\
$\mathrm{O}(21)-\mathrm{Mo}(2)-\mathrm{O}(15)$ & $106.0(2)$ & $\mathrm{O}(9)-\mathrm{P}(1)-\mathrm{O}(7)$ & $107.8(2)$ & $\mathrm{P}(4)-\mathrm{O}(1)-\mathrm{Mo}(1)$ & $132.1(2)$ \\
$\mathrm{O}(21)-\mathrm{Mo}(2)-\mathrm{O}(18)$ & $96.9(2)$ & $\mathrm{O}(2)-\mathrm{Zn}(1)-\mathrm{O}(2) \#$ & 180.0 & $\mathrm{Mo}(6)-\mathrm{O}(2)-\mathrm{Zn}(1)$ & $134.3(2)$ \\
$\mathrm{O}(21)-\mathrm{Mo}(2)-\mathrm{O}(3)$ & $97.5(2)$ & $\mathrm{O}(6) \# 1-\mathrm{Zn}(1)-\mathrm{O}(6)$ & 180.0 & $\mathrm{P}(1)-\mathrm{O}(9)-\mathrm{Zn}(2)$ & $125.9(2)$ \\
$\mathrm{O}(7)-\mathrm{Mo}(2)-\mathrm{Mo}(4)$ & $89.36(9)$ & $\mathrm{O}(4) \# 1-\mathrm{Zn}(1)-\mathrm{O}(4)$ & 180.0 & $\mathrm{P}(3)-\mathrm{O}(20)-\mathrm{Zn}(3)$ & $140.8(3)$ \\
\hline $\mathrm{Sym}$
\end{tabular}

Symmetry transformations used to generate equivalent atoms: \# 1-x, 1-y, 2-z. 
Table S2 Selected bond lengths $(\AA)$ and bond angles $\left({ }^{\circ}\right)$ of compound 2.

\begin{tabular}{llllll}
\hline $\mathrm{Mo}(1)-\mathrm{O}(17)$ & $1.686(3)$ & $\mathrm{Zn}(2)-\mathrm{O}(21)$ & $1.955(3)$ & $\mathrm{O}(9)-\mathrm{Na}(1)$ & $2.418(4)$ \\
$\mathrm{Mo}(2)-\mathrm{O}(15)$ & $1.687(3)$ & $\mathrm{Zn}(2)-\mathrm{N}(2)$ & $2.113(3)$ & $\mathrm{Na}(1)-\mathrm{O}(18) \# 3$ & $2.453(4)$ \\
$\mathrm{Mo}(3)-\mathrm{O}(14)$ & $1.683(3)$ & $\mathrm{Zn}(3)-\mathrm{O}(30)$ & $1.996(3)$ & $\mathrm{Na}(1)-\mathrm{O}(20) \# 3$ & $2.473(4)$ \\
$\mathrm{Mo}(4)-\mathrm{O}(16)$ & $1.692(3)$ & $\mathrm{P}(1)-\mathrm{O}(18)$ & $1.519(3)$ & $\mathrm{Na}(1)-\mathrm{O}(11 \mathrm{~W})$ & $2.552(8)$ \\
$\mathrm{Mo}(5)-\mathrm{O}(23)$ & $1.689(3)$ & $\mathrm{P}(2)-\mathrm{O}(21)$ & $1.507(3)$ & $\mathrm{O}(9)-\mathrm{Na}(1)$ & $2.418(4)$ \\
$\mathrm{Mo}(6)-\mathrm{O}(22)$ & $1.685(3)$ & $\mathrm{P}(3)-\mathrm{O}(29)$ & $1.507(3)$ & $\mathrm{O}(24)-\mathrm{Mo}(4) \#$ & $2.069(3)$ \\
$\mathrm{Mo}(1)-\mathrm{Mo}(4)$ & $2.6000(6)$ & $\mathrm{P}(4)-\mathrm{O}(30)$ & $1.516(3)$ & $\mathrm{O}(26)-\mathrm{Mo}(5) \#$ & $2.076(3)$ \\
$\mathrm{Zn}(1)-\mathrm{O}(4)$ & $2.146(2)$ & $\mathrm{O}(3)-\mathrm{Mo}(4) \# 1$ & $2.130(2)$ & $\mathrm{O}(28)-\mathrm{Mo}(3) \#$ & $2.066(2)$ \\
$\mathrm{O}(17)-\mathrm{Mo}(1)-\mathrm{O}(12)$ & $104.72(12)$ & $\mathrm{O}(4)-\mathrm{Mo}(2)-\mathrm{O}(25)$ & $160.31(11)$ & $\mathrm{O}(18)-\mathrm{P}(1)-\mathrm{O}(5)$ & $110.46(14)$ \\
$\mathrm{O}(17)-\mathrm{Mo}(1)-\mathrm{O}(2)$ & $102.40(12)$ & $\mathrm{O}(25)-\mathrm{Mo}(2)-\mathrm{O}(3)$ & $82.71(10)$ & $\mathrm{O}(5)-\mathrm{P}(1)-\mathrm{O}(11)$ & $107.58(13)$ \\
$\mathrm{O}(12)-\mathrm{Mo}(1)-\mathrm{O}(2)$ & $95.48(10)$ & $\mathrm{O}(15)-\mathrm{Mo}(2)-\mathrm{Mo}(3)$ & $100.08(10)$ & $\mathrm{O}(11)-\mathrm{P}(1)-\mathrm{O}(6)$ & $107.75(13)$ \\
$\mathrm{O}(12)-\mathrm{Mo}(1)-\mathrm{O}(19)$ & $86.24(11)$ & $\mathrm{O}(10)-\mathrm{Mo}(2)-\mathrm{Mo}(3)$ & $48.23(8)$ & $\mathrm{O}(30)-\mathrm{P}(4)-\mathrm{O}(31)$ & $107.0(2)$ \\
$\mathrm{O}(19)-\mathrm{Mo}(1)-\mathrm{O}(7)$ & $84.48(10)$ & $\mathrm{O}(4)-\mathrm{Mo}(2)-\mathrm{Mo}(3)$ & $49.22(7)$ & $\mathrm{Mo}(6)-\mathrm{O}(1)-\mathrm{Mo}(5)$ & $81.39(9)$ \\
$\mathrm{O}(19)-\mathrm{Mo}(1)-\mathrm{O}(5)$ & $79.28(9)$ & $\mathrm{O}(4)-\mathrm{Zn}(1)-\mathrm{O}(1)$ & $84.14(9)$ & $\mathrm{Mo}(4)-\mathrm{O}(2)-\mathrm{Mo}(1)$ & $81.75(9)$ \\
$\mathrm{O}(7)-\mathrm{Mo}(1)-\mathrm{Mo}(4)$ & $133.97(7)$ & $\mathrm{O}(4)-\mathrm{Zn}(1)-\mathrm{O}(1) \#$ & $95.86(9)$ & $\mathrm{P}(1)-\mathrm{O}(5)-\mathrm{Mo}(1)$ & $126.69(14)$ \\
$\mathrm{O}(5)-\mathrm{Mo}(1)-\mathrm{Mo}(4)$ & $88.43(6)$ & $\mathrm{O}(1)-\mathrm{Zn}(1)-\mathrm{O}(1) \#$ & $180.00(10)$ & $\mathrm{P}(1)-\mathrm{O}(5)-\mathrm{Mo}(6)$ & $126.48(13)$ \\
$\mathrm{O}(15)-\mathrm{Mo}(2)-\mathrm{O}(10)$ & $105.19(12)$ & $\mathrm{N}(2)-\mathrm{Zn}(2)-\mathrm{O}(2 \mathrm{~W})$ & $88.27(12)$ & $\mathrm{P}(2)-\mathrm{O}(19)-\mathrm{Mo}(1)$ & $136.59(16)$ \\
\hline
\end{tabular}

Symmetry transformations used to generate equivalent atoms: \# 2-x, 1-y, -z.

Table S3 Selected bond lengths $(\AA)$ and bond angles $\left({ }^{\circ}\right)$ of compound 3 .

\begin{tabular}{llllll}
\hline $\mathrm{Mo}(1)-\mathrm{O}(1)$ & $1.995(3)$ & $\mathrm{Zn}(2)-\mathrm{O}(18)$ & $1.950(5)$ & $\mathrm{P}(4)-\mathrm{O}(28)$ & $1.576(8)$ \\
$\mathrm{Mo}(1)-\mathrm{O}(4)$ & $1.977(3)$ & $\mathrm{Zn}(2)-\mathrm{N}(2)$ & $2.079(5)$ & $\mathrm{P}(3)-\mathrm{O}(25)$ & $1.501(6)$ \\
$\mathrm{Mo}(2)-\mathrm{O}(1)$ & $1.993(3)$ & $\mathrm{Zn}(3)-\mathrm{O}(4)$ & $2.034(4)$ & $\mathrm{P}(2)-\mathrm{O}(30)$ & $1.587(9)$ \\
$\mathrm{Mo}(2)-\mathrm{O}(4)$ & $1.968(5)$ & $\mathrm{P}(1)-\mathrm{O}(7)$ & $1.553(5)$ & $\mathrm{P}(1)-\mathrm{O}(9)$ & $1.552(5)$ \\
$\mathrm{Mo}(3)-\mathrm{O}(2)$ & $1.990(3)$ & $\mathrm{P}(2)-\mathrm{O}(13)$ & $1.538(5)$ & $\mathrm{O}(5)-\mathrm{Mo}(3)$ & $2.110(5)$ \\
$\mathrm{Mo}(3)-\mathrm{O}(11)$ & $1.949(6)$ & $\mathrm{P}(3)-\mathrm{O}(17)$ & $1.547(5)$ & $\mathrm{O}(21)-\mathrm{Mo}(5) \#$ & $2.053(6)$ \\
$\mathrm{Mo}(4)-\mathrm{O}(3)$ & $1.988(4)$ & $\mathrm{P}(4)-\mathrm{O}(21)$ & $1.522(6)$ & $\mathrm{O}(26)-\mathrm{Mo}(6) \#$ & $2.078(7)$ \\
$\mathrm{Zn}(1)-\mathrm{O}(1)$ & $2.163(2)$ & $\mathrm{O}(3)-\mathrm{Mo}(4) \#$ & $2.130(2)$ & $\mathrm{O}(7)-\mathrm{Mo}(5)$ & $2.305(4)$ \\
$\mathrm{O}(1)-\mathrm{Mo}(1)-\mathrm{O}(4)$ & $95.3(2)$ & $\mathrm{O}(1)-\mathrm{Mo}(2)-\mathrm{O}(4)$ & $95.7(2)$ & $\mathrm{O}(7)-\mathrm{P}(1)-\mathrm{O}(8)$ & $109.3(2)$ \\
$\mathrm{O}(1)-\mathrm{Mo}(1)-\mathrm{O}(6)$ & $86.9(2)$ & $\mathrm{O}(1)-\mathrm{Mo}(2)-\mathrm{O}(5)$ & $86.4(2)$ & $\mathrm{O}(8)-\mathrm{P}(1)-\mathrm{O}(9)$ & $108.0(2)$ \\
$\mathrm{O}(4)-\mathrm{Mo}(1)-\mathrm{O}(6)$ & $157.6(2)$ & $\mathrm{O}(4)-\mathrm{Mo}(2)-\mathrm{O}(5)$ & $158.2(2)$ & $\mathrm{O}(9)-\mathrm{P}(1)-\mathrm{O}(18)$ & $110.2(3)$ \\
$\mathrm{O}(4)-\mathrm{Mo}(1)-\mathrm{O}(8)$ & $73.2(2)$ & $\mathrm{O}(5)-\mathrm{Mo}(2)-\mathrm{O}(9)$ & $73.5(2)$ & $\mathrm{O} 13)-\mathrm{P}(2)-\mathrm{O}(15)$ & $112.1(3)$ \\
$\mathrm{O}(6)-\mathrm{Mo}(1)-\mathrm{O}(7)$ & $84.48(10)$ & $\mathrm{O}(9)-\mathrm{Mo}(2)-\mathrm{O}(12)$ & $97.0(2)$ & $\mathrm{Mo}(1)-\mathrm{O}(1)-\mathrm{Mo}(2)$ & $82.6(1)$ \\
$\mathrm{O}(6)-\mathrm{Mo}(1)-\mathrm{O}(14)$ & $83.5(2)$ & $\mathrm{O}(1)-\mathrm{Zn}(1)-\mathrm{O}(2)$ & $84.9(9)$ & $\mathrm{Mo}(3)-\mathrm{O}(2)-\mathrm{Mo}(5)$ & $81.6(1)$ \\
$\mathrm{O}(8)-\mathrm{Mo}(1)-\mathrm{Mo}(14)$ & $79.6(2)$ & $\mathrm{O}(1)-\mathrm{Zn}(1)-\mathrm{O}(3)$ & $84.7(9)$ & $\mathrm{P}(1)-\mathrm{O}(9)-\mathrm{Mo}(3)$ & $125.2(2)$ \\
$\mathrm{O}(8)-\mathrm{Mo}(1)-\mathrm{Mo}(16)$ & $170.1(2)$ & $\mathrm{O}(2)-\mathrm{Zn}(1)-\mathrm{O}(3)$ & $83.00(10)$ & $\mathrm{P}(2)-\mathrm{O}(13)-\mathrm{Mo}(3)$ & $135.3(3)$ \\
$\mathrm{O}(14)-\mathrm{Mo}(1)-\mathrm{O}(16)$ & $98.6(2)$ & $\mathrm{N}(1)-\mathrm{Zn}(3)-\mathrm{N}(2)$ & $78.4(2)$ & $\mathrm{P}(4)-\mathrm{O}(21)-\mathrm{Mo}(5)$ & $137.7(3)$ \\
\hline
\end{tabular}

Symmetry transformations used to generate equivalent atoms: \# -x, -y, 1-z. 
Table S4 BVS calculations of Mo, P and Zn centers in compounds 1-3.

\begin{tabular}{cccc}
\hline & Compound $\mathbf{1}$ & Compound & Compound $\mathbf{3}$ \\
\hline Mo1 & 5.194 & 5.106 & 5.118 \\
Mo2 & 5.174 & 5.087 & 5.087 \\
Mo3 & 5.244 & 5.114 & 5.104 \\
Mo4 & 5.140 & 5.062 & 5.106 \\
Mo5 & 5.183 & 5.094 & 5.123 \\
Mo6 & 5.219 & 5.139 & 5.072 \\
P1 & 4.739 & 4.643 & 4.668 \\
P2 & 4.819 & 4.775 & 4.912 \\
P3 & 4.799 & 4.742 & 4.727 \\
P4 & 4.819 & 4.818 & 4.803 \\
Zn1 & 1.711 & 1.722 & 1.716 \\
Zn2 & 1.767 & 1.735 & 2.032 \\
Zn3 & 2.070 & 2.006 & 2.064 \\
\hline
\end{tabular}

Table S5 Redox potentials (mV vs. Ag/ $\mathrm{AgCl}$ ) on I-I', II-II' and III-III' for 1-3 GCEs at the scan rate of $140 \mathrm{mV} \cdot \mathrm{s}^{-1}$.

\begin{tabular}{|c|c|c|c|}
\hline $\begin{array}{c}\text { Scan rate } \\
140 \mathrm{mV} \cdot \mathrm{s}^{-1}\end{array}$ & $\mathrm{E}_{\mathrm{pa}} / \mathrm{E}_{\mathrm{pc}}(\mathrm{I}) / \mathrm{mV}$ & $\mathrm{E}_{\mathrm{pa}} / \mathrm{E}_{\mathrm{pc}}(\mathrm{II}) / \mathrm{mV}$ & $\mathrm{E}_{\mathrm{pa}} / \mathrm{E}_{\mathrm{pc}}(\mathrm{III}) / \mathrm{mV}$ \\
\hline Compound $\mathbf{1}$ & $-23 /-52$ & $231 / 214$ & $370 / 339$ \\
\hline Compound $\mathbf{2}$ & $-21 /-48$ & $234 / 217$ & $371 / 334$ \\
\hline Compound $\mathbf{3}$ & $-17 /-64$ & $236 / 205$ & $386 / 315$ \\
\hline
\end{tabular}

